\title{
Caracterização magnética da Zona de Cisalhamento Lancinha no Estado do Paraná, Sul do Brasil.
}

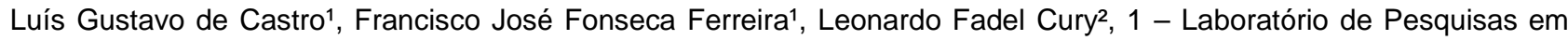
Geofísica Aplicada (LPGA/UFPR), 2 - Departamento de Geologia (DGEOL/UFPR),

Copyright 2014, SBGf - Sociedade Brasileira de Geofísica

Este texto foi preparado para a apresentação no VI Simpósio Brasileiro de Geofísica, Porto Alegre, 14 a 16 de outubro de 2014. Seu conteúdo foi revisado pelo Comitê Técnico do VI SimBGf, mas não necessariamente representa a opinião da SBGf ou de seus associados. É proibida a reprodução total ou parcial deste material para propósitos comerciais sem prévia autorização da SBGf.

\section{Abstract}

The lack of the subsurface information of the southern Ribeira Belt makes the characterization of the crossing shear zones a major challenge. Nevertheless, the influence of these structures on the installation and evolution of the Paraná Basin is widely accepted. Based on the application of methods of anomalies enhancement and depth estimates of sources, the present study aimed to the interpretation of magnetostructural framework in the area of Lancinha Shear Zone (LSZ) in the Paraná State. The results indicated the extension of the Lancinha, Morro Agudo and Itapirapuã shear zones under the Paraná Basin.

\section{Introdução}

O processamento de dados de campos potencias (gravimetria e magnetometria) é utilizado amplamente no delineamento do arcabouço estrutural de bacias sedimentares. Permite não somente o traçado de elementos de sua infraestrutura, como possibilita a estimativa da profundidade de suas fontes causativas. Contudo, a ambiguidade inerente à interpretação de dados desta natureza pode resultar em imprecisões no traçado de lineamentos. Neste sentido, é proposta uma interpretação magnética-estrutural da Zona de Cisalhamento Lancinha (ZCL), em seu segmento no Estado do Paraná, e sua continuidade sob a Bacia do Paraná, com base na integração de produtos derivados da aplicação de métodos de realce de anomalias magnéticas e de deconvolução de Euler.

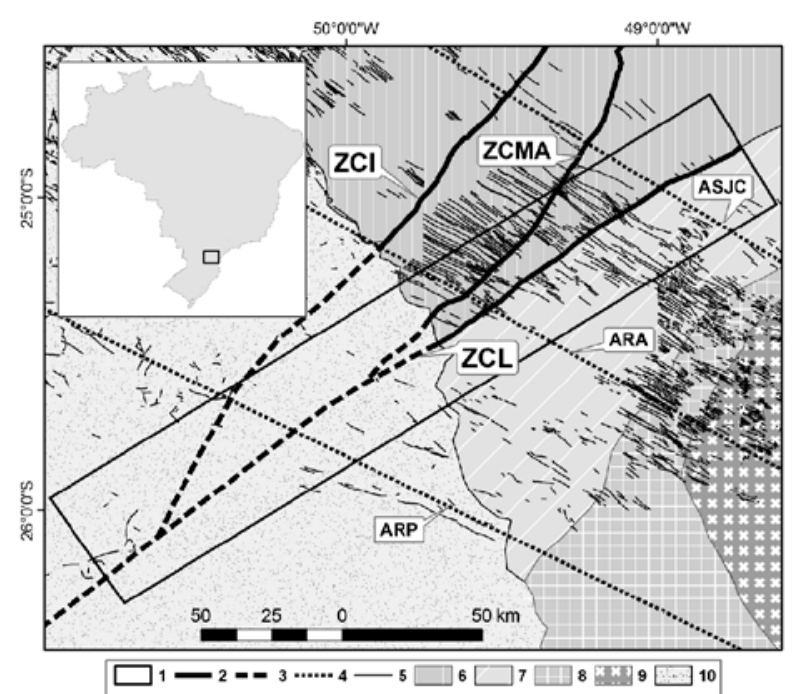

Figura 1 - Compartimentação tectono-estrutural da área de estudo e localização dos alinhamentos do Arco de Ponta Grossa. 1 - Área de estudo, 2 - Zonas de cisalhamento, 3 - Alinhamentos do Arco de Ponta Grossa (APG), 4 - Diques do APG, 5 - Divisão política; Compartimentos tectônicos: 6 - Terreno Apiaí, 7 Terreno Curitiba, 8 - Terreno Luis Alves, 9 - Terreno Paranaguá, 10 - Bacia do Paraná; Estruturas principais: ZCL - Zona de Cisalhamento Lancinha, ZCI - Zona de Cisalhamento Itapirapuã, ZCMA - Zona de Cisalhamento Morro Agudo, ASJC - Alinhamento São Jerônimo Curiúva, ARA - Alinhamento Rio Alonzo, ARP - Alinhamento Rio Piquiri.

A ZCL integra o Sistema de Falhamentos Além Paraíba-Cubatão-Lancinha, e é caracterizada como transcorrente, com movimentação predominantemente destral. No segmento estudado exibe cerca de $150 \mathrm{~km}$ de extensão em superfície, com direção média N50E.

De acordo com Sadowski (1991) a ZCL representa o segmento crustal mais superficial do sistema. Configura o principal limite tectônico entre os terrenos Apiaí (Norte) e Curitiba (Sul) (Basei et al., 1992; Campanha, 2002). Porém, a sua origem em um contexto de colisão continental não é consensual.

São destacadas duas propostas: uma que apresenta a ZCL como uma zona de transcorrência tardi-colisional correspondendo a uma tectônica de escape da convergência intercontinental; e outra que a interpreta como uma zona de sutura Neoproterozóica.

Uma avaliação inicial mostra características semelhantes entre os terrenos a Norte e a Sul da ZCL (embasamentos gnáissicos do paleopreoterozóico, metassedimentos mesoproterozóicos e granitos neoproterozóicos), porém contrastes geoquímicos, isotópicos e geocronológicos, além de particularidades na geologia do embasamento, das sequências metassedimentares e dos granitos, apontadas na bibliografia, sugerem diferenças significativas nos eventos atuantes nos terrenos Curitiba e Apiaí.

A atuação da ZCL e outras estruturas do embasamento ao longo da instalação e evolução da Bacia do Paraná foi amplamente discutida na bibliografia, porém permanecem controvérsias com relação a sua distribuição espacial e temporal.

A continuidade da ZCL sob a bacia é proposta na biliografia, com base nas interpretações de seções sísmicas, sensores remotos, mapas geofísicos e dados de campo. Os registros de atividade da ZCL são encontrados desde o Neoproterozóico até o Neógeno.

\section{Métodos}

Para o presente estudo, foram utilizados dados aeromagnéticos dos levantamentos Serra do Mar Sul e Ponta Grossa - Criciúma (Área 1), integrantes da Base de Projetos Aerogeofísicos do Brasil da CPRM (Serviço Geológico do Brasil), cuja redução ao polo foi calculada pela CPRM-PGW (2002). 
As bases reduzidas ao polo foram microniveladas e subtraídas da contribuição do IGRF (International Geomagnetic Reference Field) correspondente à data de aquisição de cada levantamento. Posteriormente, os dados do projeto Ponta Grossa-Criciúma foram continuados para cima (150 metros), e então integrados com o Projeto Serra do Mar Sul em uma base única.

Os grids resultantes foram continuados para cima (1000 metros), gerando o mapa utilizado na interpretação qualitativa.

À base de dados unificada foram aplicados os seguintes métodos de realce de anomalias: Amplitude do Sinal Analítico (ASA - Nabighian, 1972), Gradiente Horizontal Total (GHT - Cordell e Grauch, 1985), Inclinação ou Fase do Sinal Analítico (ISA - Miller e Singh, 1994), Gradiente Horizontal Total sobre a Inclinação do Sinal Analítico (GHT-ISA - Verduzco et al., 2004) e Inclinação do Sinal Analítico do Gradiente Horizontal Total (ISA-GHT - Ferreira et al., 2010, 2013).

Observou-se que as anomalias de interesse (direção NE-SW) se encontravam obliteradas pela resposta magnética do Arco de Ponta Grossa (APG). Desta forma foi aplicada uma combinação dos filtros cosseno direcional e Buterworth, na qual os sinais de alta amplitude na direção dos diques do APG (N40-60W) foram tratados como ruídos direcionais e suprimidos.

Assim, o processamento e a interpretação subsequente foram realizados paralelamente em duas bases de dados: uma sem a filtragem direcional e outra filtrada dos sinais dos diques (NW-SE).

Tendo em vista a validação da interpretação qualitativa, assim como o cálculo da profundidade das fontes causativas, foi utilizada a técnica da deconvolução de Euler (Thompson, 1982, Reid et. al., 1990).

\section{Resultados}

O mapa do campo magnético anômalo reduzido ao polo e continuado para cima (1000 metros) (CMA-RTPUW1000m) resultante do pré-processamento e da integração dos dados (Figura 2A) mostra em sua porção central a expressão magnética NW-SE dos diques de diabásio do APG.

Tal mapa constituiu a base da interpretação qualitativa, e sobre esta base foram aplicados os métodos de realce. Os mapas resultantes evidenciaram a influência das anomalias de direção NW-SE relacionadas ao APG, as quais obliteram os sinais de direção NE-SW, correspondente às estruturas de interesse desse estudo. Desta forma, foram aplicadas ao mapa CMARTP-UW1000m (Figura 2A), as filtragens para redução do sinal dos diques, resultando $n$ mapa da Figura $2 B$, no qual as estruturas de direção NE-SW são mais pronunciadas. Da mesma forma foram aplicados aos dados filtrados os métodos de realce citados, no intuito de destacar as estruturas de direção NE-SW.

Os resultados sugeriram que, tanto nos mapas com o sinal dos diques, como nos mapas filtrados, o método ISA-GHT mostrou maior eficiência do que os demais no delineamento do arcabouço magnético-estrutural, mostrando claramente a continuidade das estruturas do embasamento sob a bacia.
Assim, uma interpretação preliminar foi realizada sobre os mapas ISA-GHT com diques e filtrado, com o apoio dos demais mapas. Os resultados são exibidos nas Figuras $3 \mathrm{~A}$ e $3 \mathrm{~B}$, sobrepostos, respectivamente, aos mapas ISA-GHT e ISA-GHT filtrado. O traçado final do arcabouço magnético-estrutural da área resultou da harmonização entre as interpretações das Figuras 3A e 3B, o qual foi balizado pelas soluções de Euler (Figuras $3 C$ e 3D). A intepretação final do traçado da ZCL e das estruturas associadas, incluindo seus prolongamentos sob a Bacia do Paraná, é exibida na Figura 4.

\section{Discussão e Conclusões}

As anomalias relacionadas à ZCL na área de estudo atingem valores que variam de cerca de -40 a $78 \mathrm{nT}$ no mapa CMA-RTP-UW1000 m, chegando a atingir o intervalo de -108 a 135 nT na intersecção com o APG (Figura 3A). No mapa CMA-RTP-UW1000m filtrado dos diques (Figura 2B) os valores variam de -28 a 67 nT.

No contexto de uma colisão oblíqua, atribuída à justaposição dos terrenos Apiaí e Curitiba, a hipótese de origem e evolução da ZCL como uma sutura que evoluiu para uma zona de cisalhamento transcorrente, deveria, a princípio, ser refletida nos mapas magnéticos. As amplitudes das anomalias associadas a zonas de sutura pode variar entre 150-200 nT (Chernicoff et al., 2014) a $600 \mathrm{nT}$, ou ainda superior. Os intervalos observados nos mapas da Figura 2 são considerados baixos para caracterizar zonas de sutura preservadas.

Contudo, a ação de fluidos oxidantes, comuns em zonas de cisalhamento, poderia alterar, remobilizar ou mesmo destruir minerais magnéticos ao longo destas estruturas, reduzindo assim a amplitude das anomalias (Airo, 2002; Airo et al., 2008).

Chenicoff et al. (2014) mencionam a desmagnetização causada pela ação do cisalhamento superimposto à Zona de Sutura Cortijo, localizada na porção Sul do Cráton Rio de la Plata, onde o autor associa a desmagnetização da estrutura mais antiga pela ação hidrotermal provocada pelo cisalhamento tardio. Os autores propõem que este processo pode ocorrer em contextos de deformação progressiva, associada à orógenos de colisão oblíqua. A similaridade dos resultados obtidos por Chenicoff et al. (2014) com os padrões geofísicos observados na ZCL sugere uma proximidade dos modelos, o que explicaria as baixas amplitudes das anomalias magnéticas sobre a ZCL.

A influência da resposta do APG foi minimizada pela aplicação das filtragens, e os mapas de realce evidenciaram anomalias associadas às zonas de cisalhamento (NE-SW), tanto no contexto do PréCambriano, quanto no da Bacia do Paraná (Figura 3).

De forma especial o método ISA-GHT destacou a continuidade das estruturas sob a bacia (Figuras 3 e 4). Os resultados caracterizam o prolongamento da ZCL e de outras estruturas do embasamento, como as zonas de cisalhamento Itapirapuã (ZCl) e Morro Agudo (ZCMA), sob a Bacia do Paraná, além de sua convergência num padrão sigmoidal regional em subsuperfície (Figuras 3 e 4). A interpretação final do arcabouço magnético-estrutural foi validada pela distribuição das soluções de Euler (Figuras 3C e 3D), 
as quais indicaram que a ZCL atinge profundidades da ordem de 3000 metros no embasamento aflorante e de até 5000 metros em sua continuidade sob a Bacia do Paraná. Tanto a orientação como as profundidades de fontes indicadas pelas soluções de Euler são compatíveis com as anomalias da isoterma de Curie apresentadas por Soares et al. (2011).

\section{Agradecimentos}

Os autores agradecem a Petróleo Brasileiro S.A. (Petrobras) o financiamento da pesquisa através do Projeto Falhas, Campos de Esforços e Fluxo de Fluidos e à CPRM (Serviço Geológico do Brasil) pelos dados aeromagnéticos. L.G. de Castro e F.J.F. Ferreira agradecem a CAPES pela Bolsa de Doutorado e ao CNPq pela Bolsa de Produtividade em Pesquisa (processo $n^{\circ}$ 305810/2010-3), respectivamente.

\section{Referências}

Airo M-L, 2002. Aeromagnetic and Aeroradiometric Response to Hydrothermal Alteration. Surveys in Geophysics, 23: 273-302.

Airo M-L, Elminen T, Mertanen $S$, Niemelä R, Pajunen $M$, Wasenius $P$ \& Wennerström M. (2008). Aerogeophysical approach to ductile and brittle structures in the densely populated urban Helsinki area, southern Finland. Geological Survey of Finland, Special Paper 47, 283-308

Basei MAS, Siga Jr O, Machiavelli A, Mancini F, 1992. Evolução tectônica dos terrenos entre os Cinturões Ribeira e Dom Feliciano (PR - SC). Revista Brasileira de Geociências, 22(2): 216-221.

Campanha GAC, 2002. O papel do sistema de zonas de cisalhamento transcorrentes na configuração da porção meridional da Faixa Ribeira. Tese (Livre docência). São Paulo: Instituto de Geociências, USP.

Chernicoff CJ, Zappettini EO, Peroni J, 2014. The Ryacian El Cortijo suture zone: Aeromagnetic signature and insights for the geodynamic evolution of the southwest Rio de la Plata craton, Argentina. Geoscience Frontiers, 5: 43-52.
Cordell L \& Graunch VJS, 1985. Mapping basement magnetization zones from aeromagnetic data in the San Juan Basin, New Mexico. In: Hinze WJ (ed). The Utility of Regional Gravity and Magnetic Anomalies Maps. Society of Exploration Geophysicists, p.181-197.

CPRM-PGW, 2002. Brazil Airborne Magnetic Mapping Project (BAMMP).Technical Report and Survey Atlas. A collaboration between Paterson, Grant \& Watson Limited (PGW) and Geological Survey of Brazil (CPRM).

Ferreira FJF, Souza J, Bongiolo, ABS, Castro LG, 2013. Enhancement of the total horizontal gradient of magnetic anomalies using the tilt angle. Geophysics, 78, J33-J41.

Miller HG \& Singh V, 1994. Potential field tilt - a new concept for location of potential field sources. Journal of Applied Geophysics, 32(2-3): 213-217.

Nabighian MN, 1972. The analytic signal of twodimensional magnetic bodies with polygonal crosssection: Its properties and use for automated anomaly interpretation. Geophysics, 37(3): 507-517.

Sadowski GR, 1991. A Megafalha de Cubatão No Sudeste Brasileiro. Boletim IG-USP Serie Cientifica, 22, 15-28.

Soares, PC, Ferreira, FJF, Fiori, A. P., Castro, L. G., 2011. A proposal of new method for estimating depth of Curie surface. In: Eleventh International Congress of the Brazilian Geophysical Society, 2011, Rio de Janeiro, RJ.

Thompson DT, 1982. EULDPH: A new technique for making computer assisted depth estimates from magnetic data. Geophysics, 47(1): 31-37.

Verduzco B, Fairhead JD; Green CM; Mackenzie C, 2004. New insights into magnetic derivatives for structural mapping. The Leading Edge, 23(2): 116-119.

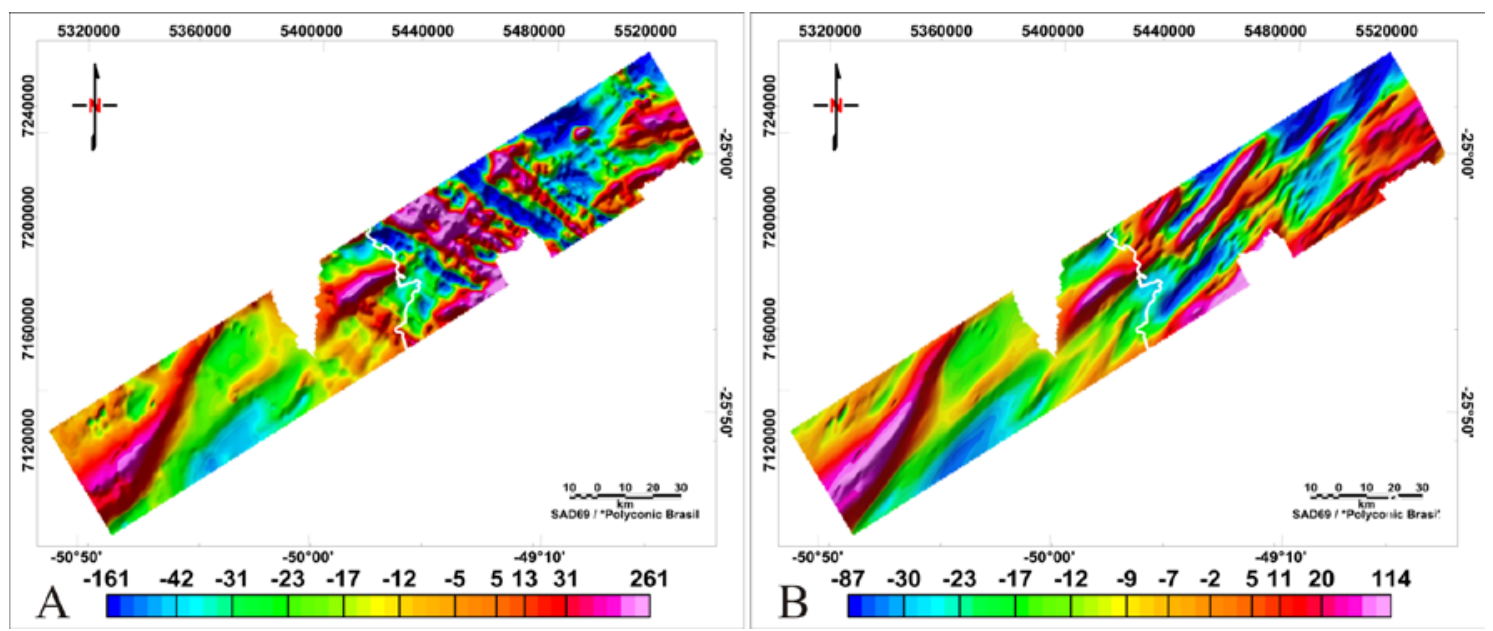

Figura 2 - (A) Mapa magnético reduzido ao polo e continuado para cima (CMA-RTP-UW1000m) e (B) CMA-RTPUW1000m filtrado dos sinais dos diques. A linha branca indica o contorno do embasamento. 


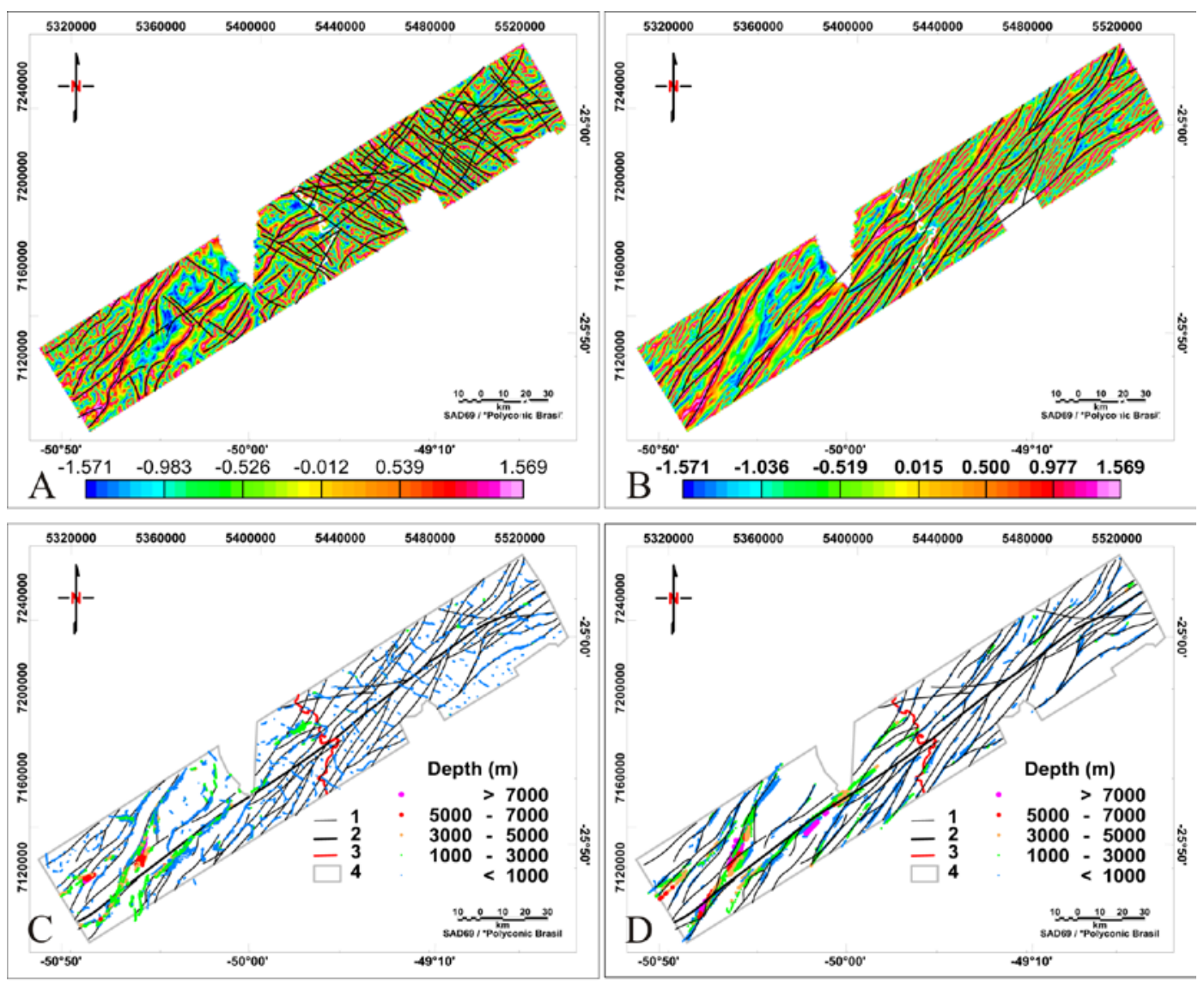

Figura 3 - (A) Interpretação magnética-estrutural sobre o mapa ISA-GHT; (B) Interpretação magnética-estrutural I dos dados filtrados dos diques sobre o ISA-GHT correspondente; (C) Mapa de soluções de Euler sobre o arcabouço magnético-estrutural; (D) Mapa do arcabouço magnético-estrutural: 1) Arcabouço magnético- estrutural, 2) Zona de Cisalhamento Lancinha, 3) Contorno do embasamento, 4) Limite dos dados.

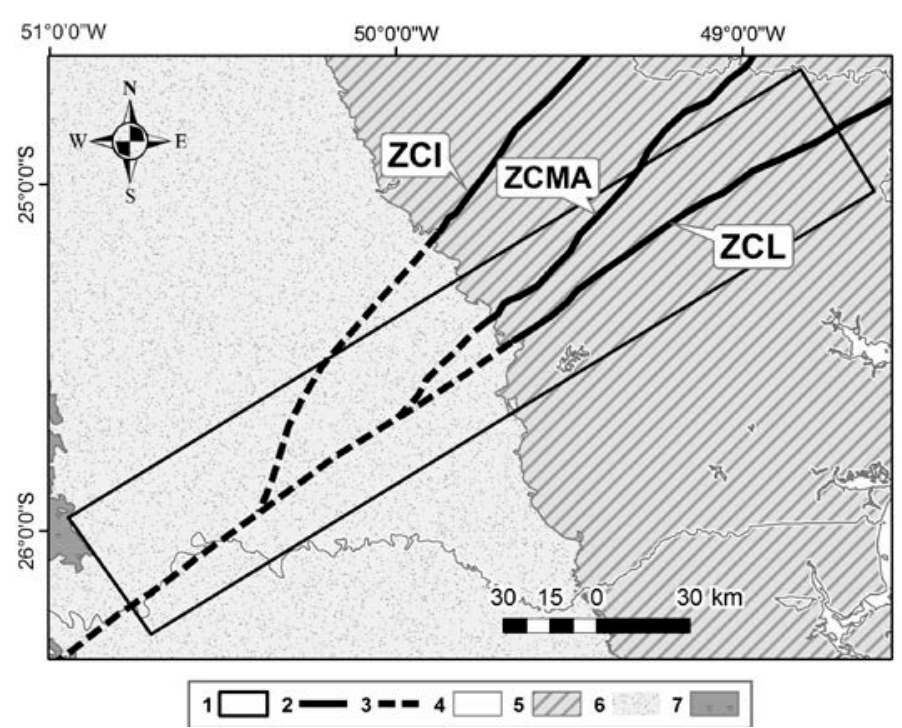

Figura 4 - Interpretação do prolongamento da ZCL e estruturas associadas sob a Bacia do Paraná: 1 - Área de estudo, 2 - Zonas de cisalhamento, 3 - Prolongamento das zonas de cisalhamento sob a bacia, 4 - Limites estaduais (SC Santa Catarina; PR - Paraná); Compartimentos Geológicos: 5 - Embasamento, 6 - Bacia do Paraná, 7 - Formação Serra Geral; Estruturas principais: ZCL - Zona de Cisalhamento Lancinha, ZCI - Zona de Cisalhamento Itapirapuã, ZCMA - Zona de Cisalhamento Morro Agudo. 